\title{
Periodontally Accelerated Osteogenic Orthodontics : A Mini Review
}

Janvi Mody ${ }^{1}$, Dishani Jain ${ }^{1}$, Shrey Mehta ${ }^{2}$, Divya Bhat ${ }^{2}$, Anjali Nagrik ${ }^{3}$, Gabriela Fernandes ${ }^{4}$

${ }^{1}$ Y.M.T. Dental College and Hospital, Kharghar, Navi Mumbai, Maharashtra, India

${ }^{2}$ Private Dental Practice, India

${ }^{3}$ Bharati Vidyapeeth Dental College, Navi Mumbai, Maharashtra, India

${ }^{4}$ Department of Oral Biology, School of dental medicine, SUNY Buffalo, Buffalo, New York, USA

Correspondence: Dr. Gabriela Fernandes, Department of Oral Biology, School of dental medicine, SUNY

Buffalo, Buffalo, New York, USA. Email: gfernand@buffalo.edu

\section{ABSTRACT}

With an increasing number of patients seeking orthodontic treatment, it often poses a challenge to the attending orthodontist to seek modalities that may promote accelerated tooth movement without compromising the underlying tissues and periodontium. Hence, PAOO (Periodontal accelerated osteogenic orthodontics was introduced in order to overcome current conventional treatment limitations. It seeks to combine selective bone corticotomy, hard tissue grafting along with the application of orthodontic forces, therefore promoting an increase in the width of the alveolar bone, lesser root resorption and lesser chair side appointments. This procedure aims at accelerating tooth movement and providing long-term improvement of the periodontium, decreased need for extractions, thereby augmenting gingival esthetics. The aim of this review was to summarize current literature regarding the role of PAOO in orthodontics.

Keywords : Periodontal Disease, Orthodontics, Wilkodontics

\section{INTRODUCTION}

The constant need for perfection is widely increasing in these recent days. Everyone wants that 'million dollar smile' and this desire has amplified in great amounts in the adult population ${ }^{1}$. Hence, they resort to orthodontic treatment. With the growing demand for this perfect smile, people simultaneously also pursue faster results ${ }^{1}$. Not only is the process of orthodontics time consuming but it also gives rise to various periodontal complications like alveolar bone resorption while uprighting ${ }^{2,3}$. It must be understood that orthodontic treatment is different for adults and adolescents. Growth factors, increased hyalinization and complications are some of the attributes to be taken into consideration.
Since there is always a solution for every problem, the hassle of adult orthodontics can also be made easier. With the help of corticotomy- assisted orthodontic treatment, more commonly known as Wilkodontics ${ }^{4,5}$, the gap between orthognathic surgery and conventional orthodontics has been successfully bridged. The technique combines the use of selective alveolar corticotomy, bone grafting and orthodontic forces ${ }^{6}$. The 'regional accelatory phenomenon' as given by Harold Frost, are a sequence of events where the osseous hard tissue is surgically wounded and this results in the reorganization of bone in the site adjacent to the injury site ${ }^{7}$. Periodontally accelerated osteogenic orthodontics works on this same phenomena.

Periodontally accelerated osteogenic orthodontics enhances the response of the periodontium to applied 
orthodontic forces, increases expansion, differential tooth movement, traction of teeth which are impacted and also the stability ${ }^{8}$. With that, it also leaves a more stable periodontal tissue with increased alveolar bulk to support the teeth, in turn leading to greater retention. The main attraction of $\mathrm{PAOO}^{7}$ is the enormous amount of time difference that it shows. On an average, where a normal orthodontic procedure would take 18-27 months, PAOO accelerates the process to a 6-9 month one ${ }^{6}$. Orthodontic braces are observed in patients undergone surgery for 3-9 months. This review paper compiles and overlooks the information regarding PAOO including its history, the mechanism behind it, its procedure, advantages, disadvantages, indications, contraindications and its technique.

\section{History}

Over these many years, there have been many studies showing the relation between selective alveolar decortication and accelerated orthodontic movement. L. C. Bryan in 1983 first described the corticotomy- facilitated tooth movement in a textbook which was introduced by Kole in $1959^{9}$ as a pathway for rapid tooth movement. Their basic principle behind this was that tooth movement is mainly resisted by cortical plates. If the continuity of the cortical plates is disrupted, the orthodontic treatment time could be reduced. As mentioned earlier, the regional acceleratory phenomenon given by Frost was one of the first findings of the osseous insult seen in long bones. A similar phenomenon was also described by Yaffe et al. ${ }^{10}$ in the mandible post periodontal flap surgery. The concept of "in vivo tissue engineering" was introduced by Murphy which described the process of morphing bone with the help of orthodontic movement in conjuction with bone activation and augmentation. Wilcko evaluated the corticotomized patients and noticed that structural integrity of the alveolar bone was lost demineralization over the root prominences. As further observed, the circumscribed corticotomy cuts on both the pressure and tension side, of the tooth were in close approximation to the apparent demineralization.

\section{Biology}

There are various indications and contraindications of this surgical treatment procedure. The basis of this procedure lies in the fact that rapid tooth movement after surgery is a consequence of the physiological and / or compositional changes of the alveolar bone. Once decortication of the bone is done, a bone graft is placed. This bone graft can be autograft, allograft or xenograft. The graft material volume is decided by various factors. Orthodontic tooth movement is carried out thereafter.

To elaborately explain it, the technique includes the surgical scaring by making vertical grooves on the labial and lingual cortical plates. On a regular basis of recall of two weeks, the patient shows significant tooth rapid tooth movement as compared to the periodontal ligament the cell mediated tooth movement. Regional Acceleratory Phenomenon, which is a part of healing event is responsible for the rapid tooth movement after which Periodontally Accelerated Osteogenic Orthodontics, ${ }^{6,711}$ is carried out. Regional Acceleratory Phenomenon shows two chief features which facilitates orthodontic tooth movement. They are namely, decreased regional bone density and accelerated bone turnover.

The various indications of PAOO are:

- Crowding

- Short span of treatment

- Retraction of canine after premolar extraction

- Need for facilitation of eruption of impacted teeth

- Molar intrusion 
- Open bite correction

- Mild class III cases

- Molar uprighting and distalisation

- Arch expansion

Contraindications are:

- Bone loss

- Periodontally compromised case

- Ankylosed teeth as a result of blunt trauma or previous luxation

- Severe class III malocclusion

- Bone diseases like rheumatoid arthritis and osteoporosis

- Root damage

- Abnormal skeletal relationship

- Intake of non-steroidal anti-inflammatory drugs and immunosuppressive drugs

The numerous advantages are:

- Speeded up treatment time- $1 / 3 r d$ time is consumed than the conventional time

- Less chances of gingival recession

- Greater retention and stability post treatment

- Less chances of root resorption

- Less chances of furcation involvement

- Repairing bone dehiscence and fenestrations as alveolar grafting is carried out.

- In the last 10 years, excellent results were seen in patients where Periodontally Accelerated Osteogenic Orthodontics was carried out.

The disadvantages are:

- Expensive procedure to carry out

- Mild surgical procedure, hence cannot be carried out in medically compromised individuals.

- Mild to moderate pain and swelling is experienced
- Proper case selection is necessary to avoid unexpected or faulty results

\section{TECHNIQUE}

To begin with, diagnostic tests should be performed and the case history of the patient should be documented. After obtaining the informed consent from the patient, the first plan of action should be scaling and root planning to rule out any other pathologies. Therefater, the surgical procedure should be performed under local anesthesia .Then, Corticotomy should be carried out in the area of the teeth to be moved. Vertical releasing incisions should then be placed from the gingival margin towards the apices of the teeth. Intracrevicular incisions should then be given to connect the buccal and lingual vertical incisions. Reflection of the mucoperiosteal flap should be done beyond the level of the apices of the teeth. Vertical grooves should then be made with the help of a round bur. Succeeding this, a horizontal groove connecting all the vertical grooves $2-3 \mathrm{~mm}$ apical to the apices of the teeth. An appropriate bioabsorbable grafting material should be placed over the decortication site. Flap repositioning along with suturing should be done along with a prescription of analgesics and antibiotics. Following the surgical procedure, orthodontic treatment should be carried out.

\section{III.CONCLUSION}

A promising surgical treatment, Periodontally Accelerated Osteogenic Orthodontics takes the branch of Orthodontics to a completely new level. It is a new age amalgamation of the use of orthodontic tooth movement with periodontal regenerative surgery. Killing the biggest limitation of Orthodontics- time, this procedure brings a new shape to the field ${ }^{7,12,13}$. With added benefits of better 
retention and stability seen post treatment, the surgical procedure proves to be a good choice for adult orthodontics where treatment is difficult due to the growth already taken place. The potential of periodontal complications are also ruled out. A boon for the older population, Periodontally Accelerated Osteogenic Orthodontics ${ }^{8,14}$ also increases the tissue turnover rate by two to three times making it a classic choice and a win-win situation for the patient, orthodontist and periodontist.

\section{REFERENCES}

[1]. Shaw WC, Addy M, Ray C. Dental and social effects of malocclusion and effectivenessof orthodontic treatment: a review. Community dentistry and oral epidemiology. 1980;8(1):36-45.

[2]. Kondo T, Hotokezaka H, Hamanaka R, et al. Types of tooth movement, bodily or tipping, do not affect the displacement of the tooth's center of resistance but do affect the alveolar bone resorption. The Angle orthodontist. 2017;87(4):563-569.

[3]. Verrusio C, Iorio-Siciliano V, Blasi A, Leuci S, Adamo D, Nicolo M. The effect of orthodontic treatment on periodontal tissue inflammation: A systematic review. Quintessence international. 2018;49(1):69-77.

[4]. Awasthi E, Sanjay K, Bhongade ML, Shrivastav S. Alveolar Bone Housing- A Modified Wilkodontics Approach- A Case Report. Journal of clinical and diagnostic research : JCDR. 2016;10(8):ZD12-15.

[5]. Kumar S, Srivastava A, Sharma A, Garg A, Kumar S. Periodontal Intervention in Speedy Orthodontics-A Case Report. Journal of clinical and diagnostic research : JCDR. 2016;10(1):ZD15-16.

[6]. Soltani L, Loomer PM, Chaar EE. A Novel Approach in Periodontally Accelerated Osteogenic Orthodontics (PAOO): A Case
Report. Clinical advances in periodontics. 2019;9(3):110-114.

[7]. Suchetha A, Lakshmi P, Prasad K, Akanksha G, Sm A, Darshan M. PAOO for faster function, aesthetics and harmony. The New York state dental journal. 2014;80(5):53-57.

[8]. Amit G, Jps K, Pankaj B, Suchinder S, Parul B. Periodontally accelerated osteogenic orthodontics (PAOO) - a review. Journal of clinical and experimental dentistry. 2012;4(5):e292-296.

[9]. Kole H. Surgical operations on the alveolar ridge to correct occlusal abnormalities. Oral surgery, oral medicine, and oral pathology. 1959;12(3):277-288 contd.

[10]. Yaffe A, Shoshan S. Re-attachment of periodontal ligament by collagen in experimentally-induced alveolar bone dehiscence in dogs. Archives of oral biology. 1987;32(2):69-73.

[11]. Munoz F, Jimenez C, Espinoza D, Vervelle A, Beugnet J, Haidar Z. Use of leukocyte and platelet-rich fibrin (L-PRF) in periodontally accelerated osteogenic orthodontics (PAOO): Clinical effects on edema and pain. Journal of clinical and experimental dentistry. 2016;8(2):e119-124.

[12]. Sanivarapu S, Addanki PK, Palaparty R, Adurty C. Periodontally accelerated osteogenic orthodontics: Novel perio-ortho interrelationship. Journal of Indian Society of Periodontology. 2018;22(5):459-462.

[13]. Brugnami F, Caiazzo A, Mehra P. Can corticotomy (with or without bone grafting) expand the limits of safe orthodontic therapy? Journal of oral biology and craniofacial research. 2018;8(1):1-6.

[14]. Vannala V, Katta A, Reddy MS, Shetty SR, Shetty RM, Khazi SS. Periodontal Accelerated Osteogenic Orthodontics Technique for Rapid Orthodontic Tooth Movement: A Systematic 
Review. Journal of pharmacy \& bioallied sciences. 2019;11(Suppl 2):S97-S106.

\section{Cite this article as :}

Janvi Mody, Dishani Jain, Shrey Mehta, Divya Bhat, Anjali Nagrik, Gabriela Fernandes, "Periodontally Accelerated Osteogenic Orthodontics : A Mini Review", International Journal of Scientific Research in Science and Technology (IJSRST), Online ISSN : 2395-602X, Print ISSN : 2395-6011, Volume 7 Issue 1, pp. 11-15, January-February 2020. Available at doi : https://doi.org/10.32628/IJSRST196666 Journal URL : http://ijsrst.com/IJSRST196666 